\title{
Curvature spectra of simple Lie groups
}

\author{
Andrzej Derdzinski · Światosław R. Gal
}

\begin{abstract}
The Killing form $\beta$ of a real (or complex) semisimple Lie group $G$ is a leftinvariant pseudo-Riemannian (or, respectively, holomorphic) Einstein metric. Let $\Omega$ denote the multiple of its curvature operator, acting on symmetric 2-tensors, with the factor chosen so that $\Omega \beta=2 \beta$. The result of Meyberg [8], describing the spectrum of $\Omega$ in complex simple Lie groups $G$, easily implies that 1 is not an eigenvalue of $\Omega$ in any real or complex simple Lie group $G$ except those locally isomorphic to $\operatorname{SU}(p, q)$, or $\operatorname{SL}(n, \mathbb{R})$, or $\operatorname{SL}(n, \mathbb{C})$ or, for even $n$ only, $\operatorname{SL}(n / 2, \mathbb{I H})$, where $p \geq q \geq 0$ and $p+q=n>2$. Due to the last conclusion, on simple Lie groups $G$ other the ones just listed, nonzero multiples of the Killing form $\beta$ are isolated among left-invariant Einstein metrics. Meyberg's theorem also allows us to understand the kernel of $\Lambda$, which is another natural operator. This in turn leads to a proof of a known, yet unpublished, fact: namely, that a semisimple real or complex Lie algebra with no simple ideals of dimension 3 is essentially determined by its Cartan three-form.
\end{abstract}

Keywords Simple Lie group · indefinite Einstein metric · left-invariant Einstein metric · Cartan three-form

Mathematics Subject Classification $17 \mathrm{~B} 20 \cdot 22 \mathrm{E} 46 \cdot 53 \mathrm{C} 30$

\section{Introduction}

Every real Lie group $G$ carries a distinguished left-invariant torsionfree connection $D$, defined by $D_{x} y=[x, y] / 2$ for all left-invariant vector fields $x$ and $y$. In view of the Jacobi identity, the curvature tensor of $D$ is $D$-parallel, and hence so is the Ricci tensor of $D$, equal

\footnotetext{
A. Derdzinski

Department of Mathematics, The Ohio State University, Columbus, OH 43210, USA

Tel.: +1-614-292-4012

Fax: +1-614-292-1479

E-mail: andrzej@math.ohio-state.edu

Ś. R. Gal

Mathematical Institute, Wrocław University, pl. Grunwaldzki 2/4, 50-384 Wrocław, Poland

E-mail: Swiatoslaw.Gal@math.uni.wroc.pl
} 
to a nonzero multiple of the Killing form $\beta$. Our convention about $\beta$ reads

$$
\beta(x, x)=\operatorname{tr}[\operatorname{Ad} x]^{2} \text { for any } x \text { in the Lie algebra } \mathfrak{g} \text { of } G .
$$

Thus, if $G$ is semisimple, $\beta$ constitutes a bi-invariant, locally symmetric, non-Ricci-flat pseudo-Riemannian Einstein metric on $G$, with the Levi-Civita connection $D$. We denote by $\Omega:\left[\mathfrak{g}^{*}\right]^{\odot 2} \rightarrow\left[\mathfrak{g}^{*}\right]^{\odot 2}$ a specific multiple of the curvature operator of the metric $\beta$, acting on symmetric symmetric bilinear forms $\sigma: \mathfrak{g} \rightarrow \mathfrak{g}$, so that, whenever $x, y \in \mathfrak{g}$,

$$
\begin{aligned}
& \text { a) }[\Omega \sigma](x, y)=2 \operatorname{tr}[(\operatorname{Ad} x)(\operatorname{Ad} y) \Sigma], \quad \text { for } \Sigma: \mathfrak{g} \rightarrow \mathfrak{g} \text { with } \\
& \text { b) } \sigma(x, y)=\beta(\Sigma x, y) .
\end{aligned}
$$

See Remark 2.4. The same formula (1.2) defines the operator $\Omega$ in a complex semisimple Lie group $G$, acting on symmetric complex-bilinear forms $\sigma$. We then identify $\Omega$ with the analogous curvature operator for the $(\mathbb{C}$-bilinear) Killing form $\beta$, treating the latter as a holomorphic Einstein metric on the underlying complex manifold of $G$.

The structure of $\Omega$ in complex simple Lie groups is known from the work of Meyberg [8], who showed that $\Omega$ is diagonalizable and described its spectrum. For the reader's convenience, we reproduce Meyberg's theorem in an appendix. His result easily leads to a similar description of the spectrum of $\Omega$ in real simple Lie algebras $\mathfrak{g}$, which we state as Theorem 4.1 and derive in Section 4 from the fact that, given any such $\mathfrak{g}$,

a) either $\mathfrak{g}$ is a real form of a complex simple Lie algebra $\mathfrak{h}$, or

b) $\mathfrak{g}$ arises by treating a complex simple Lie algebra $\mathfrak{h}$ as real.

See [6, Lemma 4 on p. 173]. The Lie-algebra isomorphism types of real simple Lie algebras $\mathfrak{g}$ thus form two disjoint classes, characterized by (1.3.a) and (1.3.b).

For both real and complex semisimple Lie groups $G$, studying $\Omega$ can be further motivated as follows. Let 'metrics' on $G$ be, by definition, pseudo-Riemannian or, respectively, holomorphic, and $\mathscr{E}$ denote the set of Levi-Civita connections of left-invariant Einstein metrics on $G$. Then, as shown in [5, Remark 12.3], whenever a semisimple Lie group $G$ has the property that 1 is not an eigenvalue of $\Omega$, the Levi-Civita connection $D$ of its Killing form $\beta$ is an isolated point of $\mathscr{E}$. The converse implication holds except when $G$ is locally isomorphic to $\mathrm{SU}(n)$, with $n \geq 3$. See [5, Theorems 22.2 and 22.3].

In a real/complex Lie algebra $\mathfrak{g}$, we define $\Lambda:\left[\mathfrak{g}^{*}\right]^{\odot 2} \rightarrow\left[\mathfrak{g}^{*}\right]^{\wedge 4}$ by

$$
(\Lambda \sigma)\left(x, y, z, z^{\prime}\right)=\sigma\left([x, y],\left[z, z^{\prime}\right]\right)+\sigma\left([y, z],\left[x, z^{\prime}\right]\right)+\sigma\left([z, x],\left[y, z^{\prime}\right]\right) .
$$

Thus, $\Lambda$ is a real/complex-linear operator, sending symmetric bilinear forms $\sigma$ on $\mathfrak{g}$ to exterior 4-forms on $\mathfrak{g}$. For the Killing form $\beta$ one has $\beta\left([x, y],\left[z, z^{\prime}\right]\right)=\beta\left([[x, y], z], z^{\prime}\right)$, as $\operatorname{Ad} z$ is $\beta$-skew-adjoint. Furthermore, by the Jacobi identity and (1.1) - (1.2.a),

$$
\text { i) } \Lambda \beta=0, \quad \text { ii) } \Omega \beta=2 \beta \text {. }
$$

If, in addition, $\mathfrak{g}$ is semisimple, there is also the operator $\Pi:\left[\mathfrak{g}^{*}\right]^{\otimes 4} \rightarrow\left[\mathfrak{g}^{*}\right]^{\otimes 2}$ with

$$
\Pi\left(\xi \otimes \xi^{\prime} \otimes \eta \otimes \eta^{\prime}\right)=\beta\left(\left[x, x^{\prime}\right], \cdot\right) \otimes \beta\left(\left[y, y^{\prime}\right], \cdot\right),
$$

for $\xi, \xi^{\prime}, \eta, \eta^{\prime} \in \mathfrak{g}^{*}$, where $x, x^{\prime}, y, y^{\prime} \in \mathfrak{g}$ are characterized by $\xi=\beta(x, \cdot), \xi^{\prime}=\beta\left(x^{\prime}, \cdot\right), \eta=$ $\beta(y, \cdot), \eta^{\prime}=\beta\left(y^{\prime}, \cdot\right)$. Formula (3.1) below shows that $\Pi\left(\left[\mathfrak{g}^{*}\right]^{\wedge 4}\right) \subset\left[\mathfrak{g}^{*}\right]^{\odot 2}$.

Our first main result, established in Section 3, relates $\Omega$ to $\Pi \Lambda:\left[\mathfrak{g}^{*}\right]^{\odot 2} \rightarrow\left[\mathfrak{g}^{*}\right]^{\odot 2}$, the composite of $\Lambda$ and the restriction of $\Pi$ to the subspace $\left[\mathfrak{g}^{*}\right]^{\wedge 4} \subset\left[\mathfrak{g}^{*}\right]^{\otimes 4}$. 
Theorem A Let $\Omega, \Lambda$ and $\Pi$ be the operators defined by (1.2), (1.4) and (1.6) for a given semisimple real/complex Lie algebra $\mathfrak{g}$. Then $2 \Pi \Lambda=-(\Omega+\mathrm{Id})(\Omega-2 \mathrm{Id})$.

Next, in Section 5, we use Meyberg's result and Theorem A to obtain the following description of $\operatorname{Ker} \Lambda$ for semisimple Lie algebras $\mathfrak{g}$. It provides a crucial step in our proof of Theorem C (see below).

Theorem B Given a real/complex semisimple Lie algebra $\mathfrak{g}$ with a direct-sum decomposition $\mathfrak{g}=\mathfrak{g}_{1} \oplus \ldots \oplus \mathfrak{g}_{s}$ into simple ideals, $s \geq 1$, let $\Lambda$ and $\Lambda_{i}$ denote the operator defined by (1.4) for $\mathfrak{g}$ and, respectively, its analog for the ith summand $\mathfrak{g}_{i}$.

(i) $\operatorname{Ker} \Lambda=\operatorname{Ker} \Lambda_{1} \oplus \ldots \oplus \operatorname{Ker} \Lambda_{s}$, where $\left[\mathfrak{g}_{i}^{*}\right]^{\odot 2} \subset\left[\mathfrak{g}^{*}\right]^{\odot 2}$ via trivial extensions.

(ii) $\Lambda=0$ if $\operatorname{dim} \mathfrak{g}=3$.

(iii) $\operatorname{dim} \operatorname{Ker} \Lambda=12$ if $\mathfrak{g}$ is simple and $\operatorname{dim} \mathfrak{g}=6$, which happens only when $\mathfrak{g}$ is real and isomorphic to the underlying real Lie algebra of $\mathfrak{s l}(2, \mathbb{C})$, while $\operatorname{Ker} \Lambda$ then consists of the real parts of all symmetric $\mathbb{C}$-bilinear functions $\mathfrak{g} \times \mathfrak{g} \rightarrow \mathbb{C}$.

(iv) $\operatorname{dim} \operatorname{Ker} \Lambda \in\{1,2\}$ whenever $\mathfrak{g}$ is simple and $\operatorname{dim} \mathfrak{g} \notin\{3,6\}$, while $\operatorname{Ker} \Lambda$ is then spanned either by the Killing form $\beta$, or by $\operatorname{Re} \beta^{\mathfrak{h}}$ and $\operatorname{Im} \beta^{\mathfrak{h}}$. The former case occurs if $\mathfrak{g}$ is complex, or real of type (1.3.a), the latter if $\mathfrak{g}$ is real of type (1.3.b), with $\beta^{\mathfrak{h}}$ denoting the Killing form of a complex simple Lie algebra $\mathfrak{h}$ in (1.3.b).

Finally, one defines the Cartan three-form $C \in\left[\mathfrak{g}^{*}\right]^{\wedge 3}$ of a Lie algebra $\mathfrak{g}$ by

$$
C=\beta([\cdot, \cdot], \cdot), \quad \text { where } \beta \text { denotes the Killing form. }
$$

The following result has been known for decades, although no published proof of it seems to exist [3]. By an isomorphism of the Cartan three-forms we mean here a vector-space isomorphism of the Lie algebras in question, sending one three-form onto the other.

Theorem $\mathbf{C}$ Let $\mathfrak{g}$ be a real/complex semisimple Lie algebra with a fixed direct-sum decomposition into simple ideals, which we briefly refer to as the "summands" of $\mathfrak{g}$.

(i) If $\mathfrak{h}$ is a real/complex Lie algebra, the Cartan three-forms of $\mathfrak{g}$ and $\mathfrak{h}$ are isomorphic and, in the real case, $\mathfrak{g}$ has no summands of dimension 3 , then $\mathfrak{h}$ is isomorphic to $\mathfrak{g}$.

(ii) If $\mathfrak{g}$ contains no summands of dimension 3 or 6 , then every automorphism of the Cartan three-form of $\mathfrak{g}$ is a Lie-algebra automorphism of $\mathfrak{g}$ followed by an operator that acts on each summand as the multiplication by a cubic root of 1 .

(iii) If $\mathfrak{g}$ is the underlying real Lie algebra of a complex simple Lie algebra and $\operatorname{dim} \mathfrak{g} \neq 6$, then every automorphism of the Cartan three-form of $\mathfrak{g}$ is complex-linear or antilinear.

Conversely, if $\mathfrak{g}$ has $k$ summands of dimension 3 and $l$ summands of dimension 6 , then the Lie-algebra automorphisms of $\mathfrak{g}$ form a subgroup of codimension $5 k+12 l$ in the automorphism group of the Cartan three-form.

We derive Theorem C from Theorem B, in Section 7.

\section{Preliminaries}

Suppose that $\mathfrak{g}$ is the underlying real Lie algebra of a complex Lie algebra $\mathfrak{h}$. We denote by $\beta$ and $C$ the Killing form and Cartan three-form of $\mathfrak{g}$, cf. (1.1) and (1.7), by $\Lambda$ the 
operator in (1.4) associated with $\mathfrak{g}$, and use the symbols $\beta^{\mathfrak{h}}, C^{\mathfrak{h}}, \Lambda^{\mathfrak{h}}$ for their counterparts corresponding to $\mathfrak{h}$. Obviously, whenever $\sigma: \mathfrak{g} \times \mathfrak{g} \rightarrow \mathbb{C}$ is a symmetric $\mathbb{C}$-bilinear form,
i) $\beta=2 \operatorname{Re} \beta^{\mathfrak{h}}$,
ii) $C=2 \operatorname{Re} C^{\mathfrak{h}}$,
iii) $\Lambda(\operatorname{Re} \sigma)=\operatorname{Re}\left(\Lambda^{\mathfrak{h}} \sigma\right)$.

For (2.1.i), see also [5, formula (13.1)]. With $\mathfrak{g}$ and $\mathfrak{h}$ as above, it is clear from (2.1.i) that

$\operatorname{Re} \beta^{\mathfrak{h}}$ and $\operatorname{Im} \beta^{\mathfrak{h}}$ span the real space of symmetric bilinear forms $\sigma$ on $\mathfrak{g}$ arising via (1.2.b) from linear endomorphisms $\Sigma$ which are complex multiples of Id.

Furthermore, (2.1.i) also implies, for dimensional reasons, that

the real parts of symmetric $\mathbb{C}$-bilinear functions $\mathfrak{g} \times \mathfrak{g} \rightarrow \mathbb{C}$ form the image under (1.2.b) of the space of $\mathbb{C}$-linear $\beta^{\mathfrak{h}}$-self-adjoint endomorphisms of $\mathfrak{h}$,

as the former space obviously contains the latter.

Let $\mathfrak{g}$ now be a Lie algebra over the scalar field $\mathbb{F}=\mathbb{R}$ or $\mathbb{F}=\mathbb{C}$. A fixed basis of $\mathfrak{g}$ allows us to represent elements $x, y$ of $\mathfrak{g}$, symmetric bilinear forms $\sigma$ on $\mathfrak{g}$, and the Lie-algebra bracket operation [,] by their components $x^{i}, y^{i}, \sigma_{i j}$ and $C_{i j}{ }^{k}$ (the structure constants of $\mathfrak{g}$ ), so that $\sigma(x, y)=\sigma_{i j} x^{i} y^{j}$ and $[x, y]^{k}=C_{i j}{ }^{k} x^{i} y^{j}$. Repeated indices are summed over. The Cartan three-form $C$ with (1.7) has the components $C_{i j k}=C_{i j}{ }^{r} \beta_{k r}$, where $\beta$ is the Killing form. The definition (1.1) of $\beta$, its bi-invariance, and the Jacobi identity now read

$$
\begin{array}{ll}
\text { i) } \beta_{i j}=C_{i p}{ }^{q} C_{j q}{ }^{p}, & \text { ii) } C_{i j k} \text { is skew-symmetric in } i, j, k, \\
\text { iii) } C_{i j}{ }^{q} C_{q k}{ }^{l}+C_{j k}{ }^{q} C_{q i}{ }^{l}+C_{k i}{ }^{q} C_{q j}{ }^{l}=0 .
\end{array}
$$

In the remainder of this section $\mathfrak{g}$ is also assumed to be semisimple. We can thus lower and raise indices using the components $\beta_{i j}$ of the Killing form $\beta$ and $\beta^{i j}$ of its reciprocal: $C_{p}^{k}{ }^{q}=\beta^{k r} C_{r p}{ }^{q}$, and $C_{j}^{s p}=\beta^{s k} C_{j k}{ }^{p}$. For any $x, y, z \in \mathfrak{g}$, one has $2 \operatorname{tr}[(\operatorname{Ad} x)(\operatorname{Ad} y)(\operatorname{Ad} z)]=$ $C(x, y, z)$, where $C$ is the Cartan three-form given by (1.7). Equivalently,

$$
2 C_{i r}^{p} C_{j q}^{r} C_{k p}^{q}=C_{i j k}
$$

In fact, by successively using the equalities $C_{p}^{k}{ }^{q}=C_{p}{ }^{q k}$ and $C_{i}{ }^{r p}=-C_{i}{ }^{p r}$ (both due to (2.4.ii)), then again (2.4.ii), (2.4.iii), and (2.4.i-ii), we get $2 C_{i r}{ }^{p} C_{j q}{ }^{r} C_{p}^{k}{ }^{q}=2 C_{i}{ }^{r p} C_{j q r} C_{p}{ }^{q k}=$ $C_{i}{ }^{r p}\left(C_{j q r} C_{p}{ }^{q k}-C_{j q p} C_{r}{ }^{q k}\right)=C_{i}{ }^{r p}\left(C_{j r}{ }^{q} C_{q p}{ }^{k}+C_{p j}{ }^{q} C_{q r}{ }^{k}\right)=-C_{i}{ }^{r p} C_{r p}{ }^{q} C_{q j}{ }^{k}=\delta_{i}^{q} C_{q j}{ }^{k}=C_{i j}{ }^{k}$. Lowering the index $k$, we obtain (2.5). Next, we introduce the linear operator

$$
T:\left[\mathfrak{g}^{*}\right]^{\otimes 2} \rightarrow\left[\mathfrak{g}^{*}\right]^{\otimes 2} \text { with }(T \sigma)_{i j}=T_{i j}^{k l} \sigma_{k l} \text {, where } T_{i j}^{k l}=2 C_{i p}{ }^{k} C_{j}^{l p} .
$$

Lemma 2.1 For $T$ and the operator $\Omega:\left[\mathfrak{g}^{*}\right]^{\odot 2} \rightarrow\left[\mathfrak{g}^{*}\right]^{\odot 2}$ given by (1.2),

(a) T leaves the subspaces $\left[\mathfrak{g}^{*}\right]^{\odot 2}$ and $\left[\mathfrak{g}^{*}\right]^{\wedge 2}$ invariant,

(b) $\Omega$ coincides with the restriction of $T$ to $\left[\mathfrak{g}^{*}\right]^{\odot 2}$.

Proof Our claim is obvious from (2.6) and the fact that, by (2.6), To is the same as $\Omega \sigma$ in (1.2), except that now $\sigma: \mathfrak{g} \times \mathfrak{g} \rightarrow \mathbb{F}$ need not be symmetric.

Lemma 2.2 For any complex simple Lie algebra $\mathfrak{g}$ and $\Omega:\left[\mathfrak{g}^{*}\right]^{\odot 2} \rightarrow\left[\mathfrak{g}^{*}\right]^{\odot 2}$ with (1.2), 
(a) $\Omega$ is diagonalizable,

(b) 2 is an eigenvalue of $\Omega$ with multiplicity 1 ,

(c) 0 is not an eigenvalue of $\Omega^{\mathfrak{h}}$,

(d) $\Omega$ has the eigenvalue 1 if and only if $\mathfrak{g}$ is isomorphic to $\mathfrak{s l}(n, \mathbb{C})$ for some $n \geq 3$.

Proof This is a special case of Meyberg's theorem, stated in the Appendix.

Remark 2.3 The isomorphism types of all complex simple Lie algebras are: $\mathfrak{s l}_{n}$, for $n \geq 2$, $\mathfrak{s p}_{n}$ (even $\left.n \geq 4\right), \mathfrak{s o}_{n}$ with $n \geq 7$, as well as $\mathfrak{g}_{2}, \mathfrak{f}_{4}, \mathfrak{e}_{6}, \mathfrak{e}_{7}$ and $\mathfrak{e}_{8}$. See [9, pp. 8 and 77].

Remark 2.4 The curvature operator of a (pseudo)Riemannian metric $\gamma$ on a manifold, acting on symmetric 2-tensors, has been studied by various authors [4], [2], [1, pp. 51-52]. It is given by $\sigma \mapsto \tau$, where $2 \tau_{i j}=\gamma^{p q} R_{i p j}{ }^{k} \sigma_{q k}$ in terms of components relative to a basis of the tangent space at any point, the sign convention about the curvature tensor $R$ being that a Euclidean tangent plane with an orthonormal basis $x, y$ has the sectional curvature $\gamma_{p q} R_{i j k}{ }^{p} x^{i} y^{j} x^{k} y^{q}$. When $\gamma$ is the Killing form $\beta$ of a semisimple Lie group $G$, treated as a left-invariant metric (see the lines following (1.1)), this operator equals $-\Omega / 16$, for $\Omega$ with (1.2). In fact, the description of the Levi-Civita connection $D$ of $\beta$ in the Introduction gives $4 R(x, y) z=[[x, y], z]$ for left-invariant vector fields $x, y, z$, that is, $4 R_{i j k}{ }^{l}=C_{i j}{ }^{p} C_{p k}{ }^{l}$. Lemma 2.1(b) now implies our claim, as $T_{i j}^{k l}=-8 \beta^{k p} R_{j p i}{ }^{l}$ due to (2.4.ii) and (2.6).

\section{Proof of Theorem A}

We use the component notation of Section 2. According to (1.4) and (1.6),

$$
\begin{aligned}
& (\Lambda \sigma)_{i j k l}=\Lambda_{i j k l}{ }^{r s} \sigma_{r s} \text { with } \Lambda_{i j k l}{ }^{r s}=C_{i j}{ }^{r} C_{k l}{ }^{s}+C_{j k}{ }^{r} C_{i l}{ }^{s}+C_{k i}{ }^{r} C_{j l}{ }^{s}, \\
& (\Pi \zeta)_{p q}=C^{i j}{ }_{p} C^{k l}{ }_{q} \zeta_{i j k l}, \quad \text { whenever } \sigma \in\left[\mathfrak{g}^{*}\right]^{\odot 2} \text { and } \zeta \in\left[\mathfrak{g}^{*}\right]^{\wedge 4} \text {. }
\end{aligned}
$$

In any real/complex semisimple Lie algebra $\mathfrak{g}$, for $C_{i j}{ }^{k}, T_{i j}^{k l}$ as in Section 2,

$$
2 C^{i j}{ }_{p} C^{k l}{ }_{q}\left(C_{i j}{ }^{r} C_{k l}{ }^{s}+C_{j k}{ }^{r} C_{i l}{ }^{s}+C_{k i}^{r} C_{j l}{ }^{s}\right)=2 \delta_{p}^{r} \delta_{q}^{s}+T_{p q}^{r s}-T_{p q}^{i k} T_{i k}^{r s} .
$$

In fact, the first of the three terms naturally arising on the left-hand side of (3.2) equals $2 \delta_{p}^{r} \delta_{q}^{s}$ since, by (2.4.i-ii), $C^{i j}{ }_{p} C_{i j}{ }^{r}=-\delta_{p}^{r}$ and $C^{k l}{ }_{q} C_{k l}{ }^{s}=-\delta_{q}^{s}$. The other two terms coincide (as skew-symmetry of $C_{p}^{i j}$ in $i, j$ gives $C^{i j}{ }_{p} C_{j k}{ }^{r} C_{i l}{ }^{s}=-C^{i j}{ }_{p} C_{i k}{ }^{r} C_{j l}{ }^{s}=C^{i j}{ }_{p} C_{k i}{ }^{r} C_{j l}{ }^{s}$ ), and so they add up to $4 C^{i j}{ }_{p} C^{k l}{ }_{q} C_{k i}{ }^{r} C_{j l}{ }^{s}$, that is, $4 C^{k l}{ }_{q} C_{j l}{ }^{s} C_{p}{ }^{j i} C_{i k}{ }^{r}=4 C^{k l}{ }_{q} C^{j}{ }_{l}^{s} C_{p j}{ }^{i} C_{i k}{ }^{r}=$ $-4 C^{k l}{ }_{q} C^{j}{ }_{l}^{s}\left(C_{j k}{ }^{i} C_{i p}{ }^{r}+C_{k p}{ }^{i} C_{i j}{ }^{r}\right)$; the rightmost equality is due to the Jacobi identity (2.4.iii). The last expression consists of the first term, $-4 C^{k l}{ }_{q} C^{j}{ }_{l}{ }^{s} C_{j k}{ }^{i} C_{i p}{ }^{r}=-4 C_{i p}{ }^{r}\left(C^{i}{ }_{k}^{j} C_{q l}{ }^{k} C^{s}{ }_{j}{ }^{l}\right)=$ $-4 C_{i p}{ }^{r} C^{i}{ }_{q}^{s}$, cf. (2.5), equal, by (2.4.ii) and (2.6), to $C_{p i}{ }^{r} C_{q}{ }^{s i}=T_{p q}^{r s}$, and the second term, $-\left(2 C_{k p}{ }^{i} C^{k l}{ }_{q}\right)\left(2 C_{i j}{ }^{r} C^{j}{ }_{l}^{s}\right)$, the two parenthesized factors of which are, for the same reasons, nothing else than $T_{p q}^{i l}$ and $T_{i l}^{r s}$. This proves (3.2).

Theorem A is now an obvious consequence of (3.1) - (3.2) and Lemma 2.1(b). 


\section{The spectrum of $\Omega$ in real simple Lie algebras}

Theorem 4.1 Let $\Omega$ denote the operator with (1.2) corresponding to a fixed real simple Lie algebra $\mathfrak{g}$, and $\Omega^{\mathfrak{h}}$ its analog for $\mathfrak{h}$, chosen so that $\mathfrak{g}$ and $\mathfrak{h}$ satisfy (1.3).

(i) $\Omega$ is always diagonalizable.

(ii) In case (1.3.a), $\Omega$ has the same spectrum as $\Omega^{\mathfrak{h}}$, including the multiplicities.

(iii) In case (1.3.b), the spectrum of $\Omega$ arises from that of $\Omega^{\mathfrak{h}}$ by doubling the original multiplicities and then including 0 as an additional eigenvalue with the required complementary multiplicity. Note that, by Lemma 2.2(c), 0 is not an eigenvalue of $\Omega^{\mathfrak{h}}$.

(iv) The eigenspace $\operatorname{Ker}(\Omega-2$ Id) is spanned in case (1.3.a) by $\beta$, and in case (1.3.b) by $\operatorname{Re} \beta^{\mathfrak{h}}$ and $\operatorname{Im} \beta^{\mathfrak{h}}$, for the Killing forms $\beta$ of $\mathfrak{g}$ and $\beta^{\mathfrak{h}}$ of $\mathfrak{h}$.

Proof By [5, Lemma 14.3(ii) and formulae (14.5) - (14.7)], if $\mathfrak{g}$ is of type (1.3.a), the complexification of $\left[\mathfrak{g}^{*}\right]^{\odot 2}$ may be naturally identified with its (complex) counterpart $\left[\mathfrak{h}^{*}\right]^{\odot 2}$ for $\mathfrak{h}$, in such a way that $\Omega^{\mathfrak{h}}$ and the Killing form $\beta^{\mathfrak{h}}$ become the unique $\mathbb{C}$-linear extensions of $\Omega$ and $\beta$. Now Lemma 2.2(a)-(b) and (1.5.ii) yield (i), (ii) and (iv) in case (1.3.a).

For $\mathfrak{g}$ of type (1.3.a), Lemma 13.1 of [5] states the following. First, $\left[\mathfrak{g}^{*}\right]^{\odot 2}$ is the direct sum of two $\Omega$-invariant subspaces: one formed by the real parts of $\mathbb{C}$-bilinear symmetric functions $\sigma: \mathfrak{h} \times \mathfrak{h} \rightarrow \mathbb{C}$, the other by the real parts of functions $\sigma: \mathfrak{h} \times \mathfrak{h} \rightarrow \mathbb{C}$ which are antilinear and Hermitian. Secondly, $\Omega$ vanishes on the "Hermitian" summand, and its action on the "symmetric" summand is equivalent, via the isomorphism $\sigma \mapsto \operatorname{Re} \sigma$, to the action of $\Omega^{\mathfrak{h}}$ on $\mathbb{C}$-bilinear symmetric functions $\sigma$. With diagonalizability of $\Omega^{\mathfrak{h}}$ again provided by Lemma 2.2(a), this proves our remaining claims. (The multiplicities are doubled since the original complex eigenspaces are viewed as real, while the eigenspace $\Omega^{\mathfrak{h}}$ for the eigenvalue 2 consists, by (v) and (1.5.ii), of complex multiples of $\beta^{\mathfrak{h}}$, the real parts of which are precisely the real linear combinations of $\operatorname{Re} \beta^{\mathfrak{h}}$ and $\operatorname{Im} \beta^{\mathfrak{h}}$.)

Remark 4.2 It is well known [9, p. 30] that, up to isomorphisms, $\mathfrak{s l}(n, \mathbb{R})$ as well as $\mathfrak{s u}(p, q)$ with $p+q=n$ and, if $n$ is even, $\mathfrak{s l}(n / 2, \mathbb{H})$, are the only real forms of $\mathfrak{s l}(n, \mathbb{C})$.

Lemma 4.3 The only complex, or real, simple Lie algebras of dimensions less than 7 are, up to isomorphisms, $\mathfrak{s l}(2, \mathbb{C})$ or, respectively, $\mathfrak{s l}(2, \mathbb{R}), \mathfrak{s u}(2), \mathfrak{s u}(1,1)$ and $\mathfrak{s l}(2, \mathbb{C})$, the last one being both complex three-dimensional and real six-dimensional. Consequently,

(i) a complex simple Lie algebra cannot be six-dimensional,

(ii) there is just one isomorphism type of a complex or, respectively, real simple Lie algebra of dimension 3 or, respectively, 6, both represented by $\mathfrak{s l}(2, \mathbb{C})$,

(iii) $\operatorname{dim} \mathfrak{g} \notin\{1,2,4,5\}$ for every real or complex simple Lie algebra $\mathfrak{g}$.

Proof According to Remark 2.3, in the complex case, only $\mathfrak{s l}(2, \mathbb{C})$ is possible. For real Lie algebras, one can use Remark 4.2 and (1.3).

Remark 4.4 We can now justify the claim, made in [5, Remark 12.3], that 1 is not an eigenvalue of $\Omega$ in any real or complex simple Lie algebra except the ones isomorphic to $\mathfrak{s l}(n, \mathbb{R}), \mathfrak{s l}(n, \mathbb{C}), \mathfrak{s u}(p, q)$ or, for even $n$ only, $\mathfrak{s l}(n / 2, \mathbb{H})$, where $n=p+q \geq 3$.

In fact, by Theorem 2.2 and parts (ii) - (iii) of Theorem 4.1, the only real or complex simple Lie algebras in which $\Omega$ has the eigenvalue 1 are, up to isomorphisms, $\mathfrak{s l}(n, \mathbb{C})$ for $n \geq 3$ and their real forms. According to Remark 4.2, these are all listed in the last paragraph. 


\section{Proof of Theorem B}

Let $\sigma \in\left[\mathfrak{g}^{*}\right]^{\odot 2}$ and $\Lambda \sigma=0$. Consequently, by (1.4), $\sigma\left([x, y],\left[z, z^{\prime}\right]\right)+\sigma\left([y, z],\left[x, z^{\prime}\right]\right)+$ $\sigma\left([z, x],\left[y, z^{\prime}\right]\right)=0$ for all $x, y, z, z^{\prime}$ in $\mathfrak{g}$. Thus, $\sigma\left([x, y],\left[z, z^{\prime}\right]\right)=0$ whenever $x, y \in \mathfrak{h}_{i}$ and $z, z^{\prime} \in \mathfrak{h}_{j}$ with $j \neq i$. The summands $\mathfrak{h}_{i}$ and $\mathfrak{h}_{j}$, being simple, are spanned by such brackets $[x, y]$ and $\left[z, z^{\prime}\right]$, and so $\mathfrak{h}_{i}$ is $\sigma$-orthogonal to $\mathfrak{h}_{j}$. As this is the case for any two summands, we obtain (i), the right-to-left inclusion being obvious. Next,

$$
\operatorname{Ker}(\Omega-2 \text { Id }) \subset \operatorname{Ker} \Lambda \subset \operatorname{Ker}(\Omega-2 \text { Id }) \oplus \operatorname{Ker}(\Omega+\text { Id }) .
$$

In fact, the second inclusion is obvious from Theorem A; the first, from Theorem 4.1(iv), (1.5.i) and (2.1.iii) applied to complex multiples $\sigma$ of $\beta^{\mathfrak{h}}$.

Part (ii) of Theorem B is immediate, as $\left[\mathfrak{g}^{*}\right]^{\wedge 4}=\{0\}$ when $\operatorname{dim} \mathfrak{g}=3$. Also, if $\mathfrak{g}$ is simple and $\operatorname{dim} \mathfrak{g}=6$, Lemma 4.3(i)-(ii) implies that $\mathfrak{g}$ is real and isomorphic to $\mathfrak{s l}(2, \mathbb{C})$. From (2.1.iii), with $\Lambda^{\mathfrak{h}} \sigma=0$ by (ii), we now get $\mathscr{F} \subset \operatorname{Ker} \Lambda$ for $\mathscr{F}=\left\{\operatorname{Re} \sigma: \sigma \in\left[\mathfrak{g}^{*}\right]^{\odot 2}\right\}$, where $\left[\mathfrak{g}^{*}\right]^{\odot 2}$ denotes the space of all symmetric $\mathbb{C}$-bilinear forms $\sigma: \mathfrak{g} \times \mathfrak{g} \rightarrow \mathbb{C}$. As $\operatorname{Re} \sigma$ uniquely determines such $\sigma$, that is, the operator $\sigma \mapsto \operatorname{Re} \sigma$ is injective, we thus have $\operatorname{dim}_{\mathbb{R}} \mathscr{F}=12$. The second inclusion in (5.1) is therefore an equality, and $\mathscr{F}=\operatorname{Ker} \Lambda$, for dimensional reasons: $\operatorname{Ker} \Lambda$ contains the subspace $\mathscr{F}$ of real dimension 12, equal, in view of part (a) of Theorem 2.2 and Theorem 4.1(iii), to $\operatorname{dim}_{\mathbb{R}}[\operatorname{Ker}(\Omega-2 \mathrm{Id}) \oplus \operatorname{Ker}(\Omega+\mathrm{Id})]$. This yields assertion (iii) in Theorem B.

Let $\mathfrak{g}$ now be simple, with $\operatorname{dim} \mathfrak{g} \notin\{3,6\}$. Due to Theorems 2.2 and 4.1(ii)-(iii), -1 is not an eigenvalue of $\Omega$. Thus, $\operatorname{Ker}(\Omega+\mathrm{Id})=\{0\}$, and the inclusions in (5.1) are equalities. In view of Theorem 4.1(iv), this completes the proof.

\section{Some needed facts from linear algebra}

In this section $\mathfrak{g}$ is the underlying real space of a finite-dimensional complex vector space $\mathfrak{h}$ and $J: \mathfrak{g} \rightarrow \mathfrak{g}$ is the operator of multiplication by $i$, also referred to as the complex structure. We denote by $\beta^{\mathfrak{h}}$ a fixed nondegenerate $\mathbb{C}$-bilinear symmetric form on $\mathfrak{h}$, so that the $\mathbb{R}$ bilinear symmetric form $\beta=2 \operatorname{Re} \beta^{\mathfrak{h}}$ on $\mathfrak{g}$ is nondegenerate as well. The same applies to any nonzero complex multiple of $\beta^{\mathfrak{h}}$. Thus, $\beta$ and $\gamma=2 \operatorname{Im} \beta^{\mathfrak{h}}$ constitute a basis of a real vector space $\mathscr{P}$ of $\mathbb{R}$-bilinear symmetric forms on $\mathfrak{g}$. All nonzero elements of $\mathscr{P}$ are nondegenerate. As $\beta^{\mathfrak{h}}$ is $\mathbb{C}$-bilinear, $\gamma(x, y)=-\beta(x, J y)$ for all $x, y \in \mathfrak{g}$. We use components relative to a basis of $\mathfrak{g}$, as in Section 2 .

Lemma 6.1 The real spaces $\mathfrak{g}$ and $\mathscr{P}$ uniquely determine the pair $\left(J, \beta^{\mathfrak{h}}\right)$ up to its replacement by $\left(J, a \beta^{\mathfrak{h}}\right)$ or $\left(-J, a \overline{\beta^{\mathfrak{h}}}\right)$, with any $a \in \mathbb{C} \backslash\{0\}$.

Proof For any basis $\kappa, \lambda$ of $\mathscr{P}$, replacing $\beta^{\mathfrak{h}}$ by a complex multiple, which leaves $\mathscr{P}$ unchanged, we assume that $\kappa=\beta$. Thus, $\lambda=u \beta+v \gamma$, where $u, v \in \mathbb{R}$ and $v \neq 0$. Writing the equality $\gamma=-\beta(\cdot, J \cdot)$ as $\gamma_{r q}=-\beta_{r s} J_{q}^{s}$, and then using the reciprocal components $\kappa^{p r}=\beta^{p r}$, we obtain $\kappa^{p r} \lambda_{r q}=\beta^{p r}\left(u \beta_{r q}-v \beta_{r s} J_{q}^{s}\right)=u \delta_{q}^{p}-v J_{q}^{p}$. Now $\pm J$ may be defined by declaring the matrix $J_{q}^{p}$ to be the traceless part of $\kappa^{p r} \lambda_{r q}$, normalized so that $J^{2}=-$ Id.

At the same time, fixing any $\kappa \in \mathscr{P} \backslash\{0\}$ we may assume, as before, that $\kappa=\beta$. Then $\kappa$ and $\gamma=-\kappa(\cdot, J \cdot)$, determine $2 \beta^{\mathfrak{h}}$, being its real and imaginary parts. Combined with the last sentence of the preceding paragraph, this completes the proof. 
The next fact concerns two mappings, rec : $\mathscr{P} \backslash\{0\} \rightarrow \mathfrak{g}^{\odot 2}$ and $\mathfrak{g}^{\odot 2} \ni \mu \mapsto \mu_{b} \in$ End $\mathfrak{g}$. The former sends every nonzero element of $\mathscr{P}$ (which, as we know, is nondegenerate) to its reciprocal. The latter is the operator of index-lowering via $\beta$, and takes values in the space of $\mathbb{R}$-linear endomorphisms of $\mathfrak{g}$, which include complex multiples of Id. We then have

$$
\left\{[\operatorname{rec}(\sigma)]_{b}: \sigma \in \mathscr{P} \backslash\{0\}\right\}=\{a \mathrm{Id}: a \in \mathbb{C} \backslash\{0\}\} .
$$

Namely, under index raising with the aid of $\beta$, the operators $A=a \mathrm{Id}$, for $a \in \mathbb{C} \backslash\{0\}$, correspond to elements $\mu$ of $\mathfrak{g}^{\odot 2}$ characterized by $\mu^{p q}=\beta^{p r} A_{r}^{q}$. Every such $\mu$ is in turn the reciprocal of $\sigma \in\left[\mathfrak{g}^{*}\right]^{\odot 2}$ defined by $\sigma_{p q}=H_{p}^{k} \beta_{k q}$, where $H=A^{-1}$ (as $\sigma_{p q} \mu^{s q}=H_{p}^{k} \beta_{k s} \beta^{s r} A_{r}^{q}=$ $H_{p}^{r} A_{r}^{q}=\delta_{p}^{q}$ ). Symmetry of $\mu$, and hence $\sigma$, is obvious from $\beta$-self-adjointness of $A$. The inverses $H$ of our operators $A=a$ Id range over nonzero complex multiples of Id as well, and so the resulting symmetric forms $\sigma$ act on $x, y \in \mathfrak{g}$ by $\sigma(x, y)=\beta(u x+v J x, y)$, where $(u, v)$ range over $\mathbb{R} \backslash\{0\}$. Therefore $\sigma=u \beta-v \gamma$, as required.

Remark 6.2 The relation $\gamma=-\beta(\cdot, J \cdot)$ for $\beta=2 \operatorname{Re} \beta^{\mathfrak{h}}$ and $\gamma=2 \operatorname{Im} \beta^{\mathfrak{h}}$ shows that, once $J$ is fixed, $\operatorname{Re} \beta^{\mathfrak{h}}$ uniquely determines $\beta^{\mathfrak{h}}$. Similarly, $\operatorname{Re} C^{\mathfrak{h}}$ and $J$ determine the Cartan three-form $C^{\mathfrak{h}}$ of a complex Lie algebra $\mathfrak{h}$, cf. (1.7). In fact, $\operatorname{Im} C^{\mathfrak{h}}=-\operatorname{Re} C^{\mathfrak{h}}(\cdot, \cdot, J \cdot)$.

Remark 6.3 The bracket [,] of a real/complex semisimple Lie algebra is uniquely determined by $C$ and $\beta$ via (1.7). Knowing $C$ and the set of nonzero scalar multiples of $\beta$, rather than $\beta$ itself, makes [,] unique up to multiplications by cubic roots of 1 . Such factors must be allowed as multiplying [,] by a scalar $r$ replaces $\beta$ and $C$ with $r^{2} \beta$ and $r^{3} C$.

Remark 6.4 In the first sentence of Remark 6.3, treating $C$ and $\beta$ formally, we see that in the complex case $\bar{C}$ and $\bar{\beta}$ determine, via (1.7), the same bracket [,] as $C$ and $\beta$.

\section{Proof of Theorem C}

For a real/complex Lie algebra $\mathfrak{g}$, let the mapping $\Phi:\left[\mathfrak{g}^{*}\right]^{\wedge 3} \times \mathfrak{g}^{\odot 2} \rightarrow\left[\mathfrak{g}^{*}\right]^{\wedge 4}$ be defined by $[\Phi(C, \mu)]\left(x, y, z, z^{\prime}\right)=\mu\left(C(x, y), C\left(z, z^{\prime}\right)\right)+\mu\left(C(y, z), C\left(x, z^{\prime}\right)\right)+\mu\left(C(z, x), C\left(y, z^{\prime}\right)\right)$, where $\mu \in \mathfrak{g}^{\odot 2}$ is treated as a symmetric real/complex-bilinear form on $\mathfrak{g}^{*}$, and $C(x, y)$ stands for the element $C(x, y, \cdot)$ of $\mathfrak{g}^{*}$. If $\mathfrak{g}$ is also semisimple, the isomorphic identification $\mathfrak{g} \approx \mathfrak{g}^{*}$ provided by the Killing form $\beta$ induces an isomorphism $\left[\mathfrak{g}^{*}\right]^{\odot 2} \rightarrow \mathfrak{g}^{\odot 2}$, which we write as $\sigma \mapsto \sigma^{\sharp}$. Then, in view of (1.4) and (1.7),

$$
\Phi\left(C, \sigma^{\sharp}\right)=\Lambda \sigma \quad \text { for any } \sigma \in \mathfrak{g}^{\odot 2} \text { and the Cartan three-form } C .
$$

Theorem $\mathrm{C}$ is a trivial consequence of the following result combined with Lemma 4.3(ii) and the fact that, by multiplying a Lie-algebra bracket operation [,] by a nonzero scalar, one obtains a Lie-algebra structure isomorphic to the original one.

Lemma 7.1 In a real or complex semisimple Lie algebra $\mathfrak{g}$, the Cartan three-form and the vector-space structure of $\mathfrak{g}$ uniquely determine each of the following objects.

(a) The vector subspaces constituting the simple direct summand ideals of $\mathfrak{g}$.

(b) Up to a sign, in the real case, the complex structure, defined as in Section 6, of every summand ideal $\mathfrak{g}^{\prime}$ with $\operatorname{dim}_{\mathbb{R}} \mathfrak{g}^{\prime} \neq 6$ which is a complex Lie algebra, treated as real.

(c) Up to multiplications by cubic roots of 1 , the restrictions of the Lie-algebra bracket of $\mathfrak{g}$ to all such summands of dimensions other than 3 or 6 . 
(d) The Lie-algebra isomorphism types of all summand ideals $\mathfrak{g}^{\prime}$ with $\operatorname{dim}_{\mathbb{R}} \mathfrak{g}^{\prime} \neq 3$.

Proof Let $C$ be the Cartan three-form of $\mathfrak{g}$. By (7.1), Ker $\Delta=\left\{\sigma^{\sharp}: \sigma \in \operatorname{Ker} \Lambda\right\}$ for the real /complex-linear operator $\Delta: \mathfrak{g}^{\odot 2} \rightarrow\left[\mathfrak{g}^{*}\right]^{\wedge 4}$ given by $\Delta \mu=\Phi(C, \mu)$. Then, if one views all $\mu \in \operatorname{Ker} \Delta \subset \mathfrak{g}^{\odot 2}$ as linear operators $\mu: \mathfrak{g}^{*} \rightarrow \mathfrak{g}$,

(e) the simple direct summands of $\mathfrak{g}$ are precisely the minimal elements, in the sense of inclusion, of the set $\mathbf{S}=\left\{\mu\left(\mathfrak{g}^{*}\right): \mu \in \operatorname{Ker} \Delta\right.$, and $\operatorname{dim} \mu\left(\mathfrak{g}^{*}\right)=3$ or $\left.\operatorname{dim} \mu\left(\mathfrak{g}^{*}\right) \geq 6\right\}$.

In fact, $\mathbf{S}$ consists of the images of those linear endomorphisms $\Sigma: \mathfrak{g} \rightarrow \mathfrak{g}$ which correspond via (1.2.b) to elements $\sigma$ of $\operatorname{Ker} \Lambda$, and have $\operatorname{rank} \Sigma \notin\{0,1,2,4,5\}$. To describe all such $\Sigma$, we use the four parts of Theorem B, referring to them as (i) - (iv). Specifically, by (i), our $\Sigma$ are direct sums of linear endomorphisms $\Sigma_{i}$ of the simple direct summands $\mathfrak{g}_{i}$ of $\mathfrak{g}$, while $\Sigma_{i}$ are themselves subject to just two restrictions: one due to the exclusion of ranks $0,1,2,4$ and 5 , the other depending, in view of (ii) - (iv), on $d_{i}=\operatorname{dim} \mathfrak{g}_{i}$, as follows. If $d_{i}=3$, (ii) states that $\Sigma_{i}$ is only required to be $\beta$-self-adjoint (to reflect symmetry of $\sigma_{i}$ related to $\Sigma_{i}$ as in (1.2.b)). Similarly, it is clear from (iv) and (2.2) that, with a specific the scalar field $\mathbb{F}$,

$\Sigma_{i}$ is a nonzero $\mathbb{F}$-multiple of Id when $d_{i} \notin\{3,6\}$, where $\mathbb{F}=\mathbb{C}$ if $\mathfrak{g}_{i}$ is either complex or real of type (1.3.b), and $\mathbb{F}=\mathbb{R}$ for real $\mathfrak{g}_{i}$ of type (1.3.a).

In the remaining case, $d_{i}=6$. Then, by (iii) and Theorem B(iii), $\Sigma_{i}$ is complex-linear and $\beta$-self-adjoint, cf. (2.3) and (2.1.i), but otherwise arbitrary.

The image $\Sigma(\mathfrak{g})$ of any $\Sigma$ as above is the direct sum of the images of its summands $\Sigma_{i}$, and so it can be minimal only if there exists just one $i$ with $\Sigma_{i} \neq 0$. For this $i$, minimality of $\Sigma(\mathfrak{g})=\Sigma_{i}\left(\mathfrak{g}_{i}\right)$ implies that $\Sigma(\mathfrak{g})=\mathfrak{g}_{i}$. In fact, in view of the last paragraph, the cases $d_{i}=3$ and $d_{i} \notin\{3,6\}$ are obvious (the former since rank $\Sigma_{i} \geq 3$ ) while, if $d_{i}=6$, complex-linearity of $\Sigma_{i}$ precludes not just $0,1,2,4$ and 5 , but also 3 from being its real rank.

We thus obtain one of the inclusions claimed in (e): every minimal element of $\mathbf{S}$ equals some summand $\mathfrak{g}_{i}$. Conversely, any fixed summand $\mathfrak{g}_{i}$ is an element of $\mathbf{S}$, realized by $\Sigma$ with $\Sigma_{i}=\operatorname{Id}$ and $\Sigma_{j}=0$ for all $j \neq i$, cf. Lemma 4.3(iii). Minimality of $\mathfrak{g}_{i}$ is in turn obvious from (7.2) if $d_{i} \notin\{3,6\}$, while for $d_{i}=3$ or $d_{i}=6$ it follows from the restriction on $\operatorname{rank} \Sigma$ combined, in the latter case, with complex-linearity of $\Sigma_{i}$. This yields (e).

Now (a) is obvious from (e), as $\Delta$ and $\mathbf{S}$ depend only on $C$ and the vector-space structure of $\mathfrak{g}$. To prove (b) - (c), we fix $i$ with $d_{i} \notin\{3,6\}$. Elements $\mu$ of Ker $\Delta$ having $\mu\left(\mathfrak{g}^{*}\right)=\mathfrak{g}_{i}$ correspond, via (1.2.b) followed by the assignment $\sigma \mapsto \mu=\sigma^{\sharp}$, to endomorphisms $\Sigma$ of $\mathfrak{g}$ which satisfy (7.2) and vanish on $\mathfrak{g}_{j}$ for $j \neq i$. Any such $\mu$, now viewed as a bilinear form on $\mathfrak{g}^{*}$, is therefore obtained from a bilinear form $\mu_{i}$ on $\mathfrak{g}_{i}^{*}$ by the trivial extension to $\mathfrak{g}^{*}$, that is, pullback under the obvious restriction operator $\mathfrak{g}^{*} \rightarrow \mathfrak{g}_{i}^{*}$.

If $\mathbb{F}=\mathbb{R}$, it is immediate from (7.2) that the resulting forms $\mu_{i}$ are nonzero multiples of the reciprocal of the Killing form of $\mathfrak{g}_{i}$, and Remark 6.3 implies (c). Next, let $\mathbb{F}=\mathbb{C}$. We denote $\mathfrak{g}_{i}$ treated as a complex Lie algebra by $\mathfrak{h}$ and Cartan three-form of $\mathfrak{h}$ by $C^{\mathfrak{h}}$. Formula (6.1) states that, in view of (7.2), the reciprocals of our $\mu_{i}$ are precisely the nonzero elements of the space $\mathscr{P}$ defined in Section 6. Thus, Lemma 6.1, (2.1.ii) and Remark 6.2 imply that $C$ determines the triple $\left(J, \beta^{\mathfrak{h}}, C^{\mathfrak{h}}\right)$ uniquely up to replacements by $\left(J, a \beta^{\mathfrak{h}}, a C^{\mathfrak{h}}\right)$ or $\left(-J, a \overline{\beta^{\mathfrak{h}}}, a \overline{C^{\mathfrak{h}}}\right)$, with $a \in \mathbb{C} \backslash\{0\}$. This proves (b), while using Remarks $6.3-6.4$ we obtain (c) for $\mathbb{F}=\mathbb{C}$ as well. Finally, (c) and Lemma 4.3(i)-(iii) easily yield (d). 


\section{Appendix: Meyberg's theorem}

For any complex simple Lie algebra $\mathfrak{g}$, the operator $\Omega$ with (1.2) is diagonalizable. Its systems Spec $[\mathfrak{g}]$ of eigenvalues and Mult $[\mathfrak{g}]$ of the corresponding multiplicities are

$$
\begin{aligned}
\operatorname{Spec}\left[\mathfrak{s l}_{n}\right] & =(2,1,2 / n,-2 / n) \text { and } \\
\operatorname{Mult}\left[\mathfrak{s l}_{n}\right] & =\left(1, n^{2}-1, n^{2}(n-3)(n+1) / 4, n^{2}(n+3)(n-1) / 4\right), \text { if } n \geq 4 . \\
\operatorname{Spec}\left[\mathfrak{s p}_{n}\right] & =(2,(n+4) /(n+2),-4 /(n+2), 2 /(n+2)) \text { for even } n \geq 4, \text { and } \\
\operatorname{Mult}\left[\mathfrak{s p}_{n}\right] & =(1,(n-2)(n+1) / 2, n(n+1)(n+2)(n+3) / 24, n(n-1)(n-2)(n+3) / 12) . \\
\operatorname{Spec}\left[\mathfrak{s o}_{n}\right] & =(2,(n-4) /(n-2), 4 /(n-2),-2 /(n-2)) \text { if } n=7 \text { or } n \geq 9, \text { while } \\
\operatorname{Mult}\left[\mathfrak{s o}_{n}\right] & =(1,(n+2)(n-1) / 2, n(n-1)(n-2)(n-3) / 24, n(n+1)(n+2)(n-3) / 12)
\end{aligned}
$$

and, if $\mathfrak{g}$ is one of the exceptional complex Lie algebras $\mathfrak{s l}_{2}, \mathfrak{s l}_{3}, \mathfrak{g}_{2}, \mathfrak{s o}_{8}, \mathfrak{f}_{4}, \mathfrak{e}_{6}, \mathfrak{e}_{6}, \mathfrak{e}_{7}, \mathfrak{e}_{8}$

$$
\operatorname{Spec}[\mathfrak{g}]=(2,(1+w) / 6,(1-w) / 6) \text {, with Mult }[\mathfrak{g}] \text { equal to }
$$

$$
(1,3 d[(d+2) w-(d+32)] /[w(11-w)], 3 d[(d+2) w+(d+32)] /[w(11+w)]),
$$

where $d=\operatorname{dim} \mathfrak{g}$ and $w=[(d+242) /(d+2)]^{1 / 2}$. This is a result of Meyberg [8] who, instead of our $\Omega$, studied the operator $T=\Omega / 2$. (The exponent $1 / 2$ is missing in [8]). For $\mathfrak{s h}_{2}$, the resulting "eigenvalue" $4 / 3$ of multiplicity 0 should be disregarded. All isomorphism types of complex simple Lie algebras are listed above, cf. Remark 2.3.

The dimensions $d$ of $\mathfrak{s l}_{2}, \mathfrak{s l}_{3}, \mathfrak{g}_{2}, \mathfrak{s o}_{8}, \mathfrak{f}_{4}, \mathfrak{e}_{6}, \mathfrak{e}_{6}, \mathfrak{e}_{7}, \mathfrak{e}_{8}$ are $3,8,14,28,52,78,133,248$ [7, p. 21]. The eigenvalues 0 and 1 in (7.3) would correspond to $w=1$ or $w=5$, of which only the latter occurs, for $d=8$ and $\mathfrak{g}=\mathfrak{s l}_{3}$, in agreement with Lemma 2.2.

\section{References}

1. Besse, A.L.: Einstein Manifolds. Ergeb. Math. Grenzgeb. (3), vol. 10. Springer, Berlin (1987)

2. Bourguignon, J.P., Karcher, H.: Curvature operators: pinching estimates and geometric examples. Ann. Sci. École Norm. Sup. (4) 11, 71-92 (1978)

3. R. Bryant, private communication (2009)

4. Calabi, E., Vesentini, E.: On compact, locally symmetric Kähler manifolds. Ann. of Math. (2) 71, 472507 (1960)

5. Derdzinski, A., Gal, Ś.R.: Indefinite Einstein metrics on simple Lie groups. Indiana Univ. Math. J. (to appear). arXiv:1209.6084v2

6. Hausner, M., Schwartz, J.T.: Lie Groups, Lie Algebras. Gordon and Breach, New York (1968)

7. Iachello, F.: Lie Algebras and Applications. Lecture Notes in Physics, vol. 708. Springer, Berlin (2006)

8. Meyberg, K.: Spurformeln in einfachen Lie-Algebren. Abh. Math. Sem. Univ. Hamburg 54, 177-189 (1984)

9. Onishchik, A.L.: Lectures on Real Semisimple Lie Algebras and their Representations. ESI Lectures in Mathematics and Physics, EMS, Zürich (2004) 